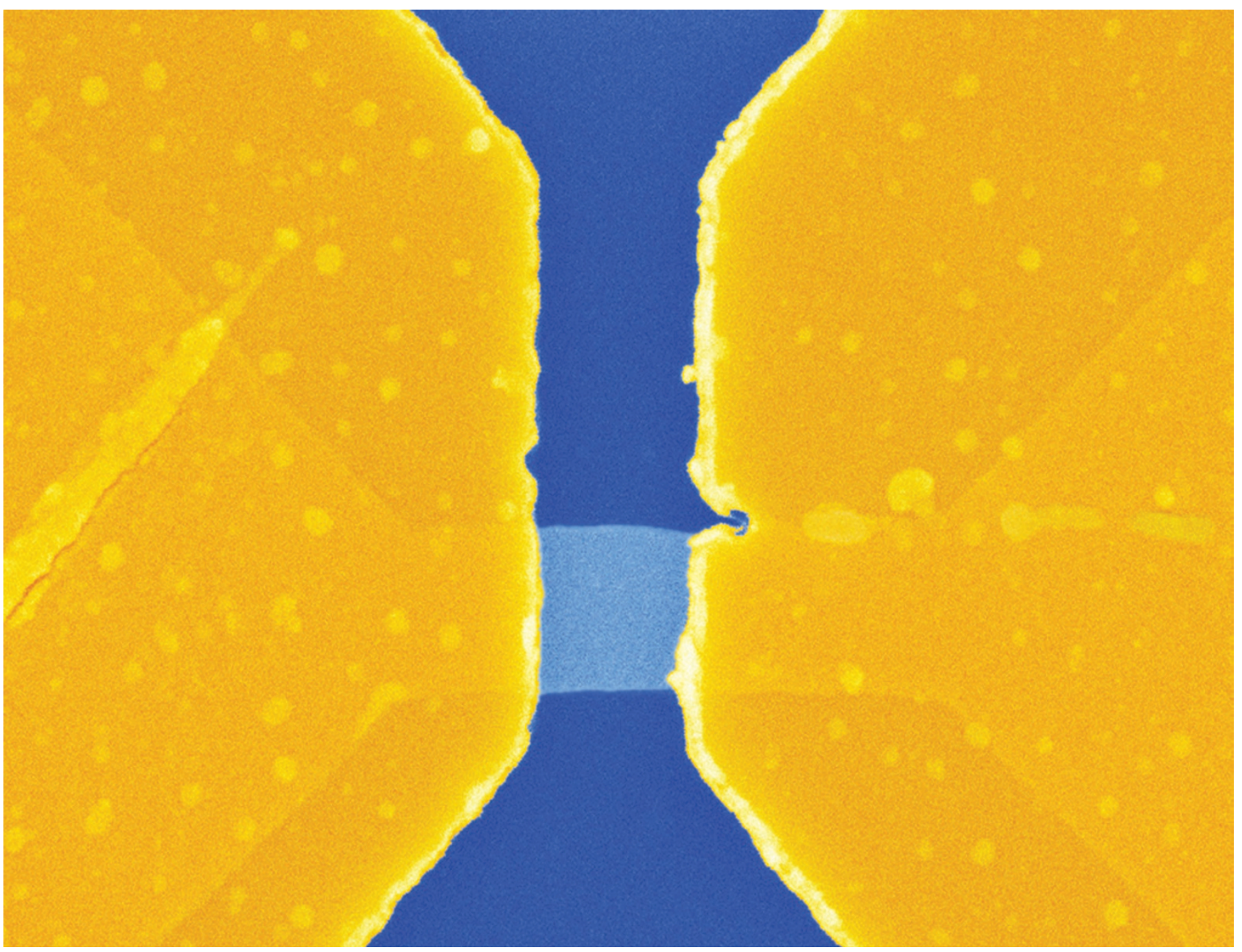

Coloured scanning electron micrograph of a transistor composed of a graphene wire (centre), gold electrodes (dark yellow) and silicon (blue).

\title{
ELECTRONICS
}

\section{Back to analogue}

\section{Trying to shoehorn graphene into a digital circuit isn't working. But there may be another potential path to glory.}

\section{BY KATHERINE BOURZAC}

$\mathrm{I}$ n July 2008, Tomás Palacios was sitting in an airport in Boston, Massachusetts, waiting for a delayed flight and thinking about his latest batch of graphene transistors. Yet again, they weren't working as they should. Palacios was excited when he learned about graphene only four years before, and he hoped this form of carbon could hasten a future generation of computer chips once silicon was pushed to its physical limits. But a major hurdle blocks the way: there seems to be no good way to make graphene transistors that can perform the on-off digital logic that is the basis of computation. For the past few years, many researchers have been tinkering with graphene to make it behave more like a conventional material. Like Palacios, many had been disappointed. "It is very difficult to fight against nature," he says.

With hours to kill at the airport, Palacios started to mull over his results. Studying some of his latest current-versus-voltage graphs, he realized that the electrical behaviour of a single graphene transistor looks like that of a circuit comprising multiple silicon ones. He called one of his students and asked him to test a single graphene transistor against the silicon circuit. When he finally got back to his lab at the Massachusetts Institute of Technology in Cambridge, he saw that the graphene transistor outperformed the silicon grouping - and that his group had just created a new branch of research.

\section{NANOSPAGHETTI}

Ever since Andre Geim and Konstantin Novoselov of the University of Manchester, UK, published their seminal Science paper in 2004 describing graphene's electrical properties, researchers have been trying to figure out what to do with the material. Some of this effort has been like trying to push a square peg into a round hole. "If you try to fit graphene into a 
silicon box, it's a bit awkward," says James Tour, a chemist at Rice University in Houston, Texas. Now, many people are testing graphene in transistors to see what it does well, which might not be digital computing. They're shifting their focus towards speedier flexible electronics, more compact and efficient circuits that operate in unusual ways, and high-performance analogue electronics that could improve telecommunications and mobile data streaming.

The faster that electrons can move from one end of a transistor material to the other - that is, the higher its mobility - the faster that information can move around a computer chip. When Geim and Novoselov wired up flakes of graphene, they found that electrons sailed swiftly through them. Indeed, electrons in graphene behave as if they have no mass. Graphene's mobility at room temperature can be as high as 100,000 square centimetres per volt per second - some 70 times higher than it is for silicon.

In addition to speed, graphene offers a potential size advantage. Consisting of a single layer of carbon atoms, the material is effectively twodimensional. This means it should be possible to make computer switches even smaller than the silicon transistors that form the backbone of the US\$250-billion semiconductor market. "We would like to find a new switch, a material to replace silicon," says Luigi Colombo, a researcher at Texas Instruments in Dallas.

Graphene might also be easy to manufacture. Fengnian Xia, a researcher at the IBM Watson Research Center in Yorktown Heights, New York, says his lab can already grow sheets of the material on the silicon wafers that the semiconductor industry uses for large-scale chip manufacturing. Compatibility with existing infrastructure is a big plus of graphene over other new materials. Carbon nanotubes, for example, have similar properties to graphene but fail the infrastructure test: they are difficult to align on a surface, tending to tangle up like spaghetti.

\section{MIND THE GAP}

The big problem with making digital switches from graphene is that the material lacks an electrical property called a bandgap - essential for digital logic. To understand this phenomenon, it's helpful to look at a conventional silicon transistor, the type found in the hundreds of millions of today's computer chips. These transistors have four main parts, a silicon channel and three metal terminals: gate, source and drain. An electric voltage applied at the gate controls the flow of current from the source electrode through the silicon channel to the drain electrode. Silicon has certain energy states where it's conductive, and

ONATURE.COM For some of the latest research on graphene transistors: go.nature.com/2d2ovl

through the gate, it's possible to switch silicon's energy level between the conductive state, to turn the transistor on and make a 1 , and the insulating state, to turn the transistor off and make a 0 . Getting that zero is critical for efficient digital logic, and to keep power consumption in check. Because there are hundreds of millions of transistors in a microprocessor, it's important to be able to turn them off when they're not in use, otherwise their power requirements would be unsustainable. But graphene does not naturally have a bandgap and can't make a zero. Researchers have developed ways to overcome this problem. Graphene has been doped with chemicals, punched with tiny holes, and exposed to all kinds of abuse to induce a bandgap.

Unfortunately, such interventions also compromise graphene's charge mobility, so the resulting transistors are not fast enough to be worth the bother. "Nobody has made a switch out of graphene that is technologically feasible and comparable to

"We knew from the beginning it would be challenging to make graphene work for logic applications." silicon," says Walter de Heer, a physicist at the Georgia Institute of Technology in Atlanta, who made some of the first graphene transistors. Novoselov agrees, but notes that people are still coming to grips with graphene. "We knew from the beginning that it would be challenging to make graphene work for logic applications," he says.

So researchers are now using graphene transistors in a field that plays better to its strengths: analogue electronics. Analogue devices, the electronics at work in communications equipment such as receivers, transmitters and amplifiers, don't speak in zeros and ones but operate over a range of voltages. Wherever an electronic device meets the outside world, there are analogue electronics. The chip board in a smartphone has many. Besides processing audio, it handles photographs taken with the camera, while other circuits stream video. So although much of what we think of as information processing now occurs in the digital realm, "analogue sets the speed limit on data", says electrical engineer Deji Akinwande, who studies carbon nanomaterials at the University of Texas at Austin.

\section{SPEEDING COMMUNICATIONS}

Phaedon Avouris, a materials scientist at IBM, has been working on graphene transistors since 2006. Working independently, his group and Philip Kim's at Columbia University in New York made the first graphene transistors with a bandgap by slicing the material into ribbons tens of atoms wide with the help of an electron beam. The procedure is complicated, and the resulting transistors are slow. "It became clear that this will not go too far in a practical application," he says. "If graphene is really the miracle material, use it as it is and don't try to change it into something else."

For Avouris, using graphene properly means taking advantage of its high mobility in highfrequency analogue electronics and its ability to integrate well with other materials. Generally, the faster the analogue electronics can switch - the higher their frequency - the less noisy, or error-prone, they are. Higher-frequency electronics can produce stronger signals that can travel farther, and they use less energy than low-frequency electronics. And signals sent at higher frequencies have lower interference, which means more of them can be nestled together in a given band of the spectrum for faster data transmission.

The analogue electronics in consumer goods are, like digital electronics, based on silicon. For niche applications, such as encoding military messages, and transmitting and receiving signals in high-power mobile-phone base stations, speedier electronics made from more expensive materials are required. These so-called 'III-V' materials (named after those two groups in the periodic table their elements come from) include gallium arsenide and indium phosphide. Some III-V transistors operate at frequencies as high as 1 terahertz (1,000 gigahertz). But whereas III-V materials aren't compatible with silicon electronics and need to be placed on their own chips, graphene is compatible with silicon.

So far, no graphene circuits have reached terahertz speeds. In 2010, Avouris made transistors with speeds of $100 \mathrm{GHz}$ - about ten times as fast as the best silicon transistors. Avouris believes the only way forward is to use the equipment already on the floor of today's semiconductor factories, so his group has been mostly using graphene films on the surface of the same wafers used to make computer chips. The IBM result has been overtaken by Xiangfeng Duan, a chemist at the University of California, Los Angeles, with a $300-\mathrm{GHz}$ graphene transistor made with graphene flakes. But these flakes are not as manufacturing-friendly as the films of graphene that the IBM group used. Both groups achieved these speeds by taking good care not to damage the graphene - a tough challenge because other materials must be laid on the graphene in order to make a transistor.

Electronics require integrated circuits rather than single transistors. During the summer of 2011, Avouris' team made the first graphene integrated circuit on a wafer, reaching a record speed of about $10 \mathrm{GHz}$. The circuit combined a graphene transistor with two inductors to make one of the workhorse devices of telecommunications: a frequency mixer. Frequency mixers combine a signal to be transmitted - say, the sound of a voice as picked up by a mobile-phone microphone - with a reference signal so that it can travel through the air. Frequency mixers also unscramble such signals at the other end. Similar efforts to develop 


\section{READY TO GO}

One of the advantages of graphene electronics is that they can be manufactured using equipment and processes already used by the chip industry.

1. The 8-inch wafer is covered in graphene transistors.

2. The wafer can be cut into chips.

3. Microscope zooms in on a single transistor.

4. View of the gates (horizontal bars) that control the transistor.
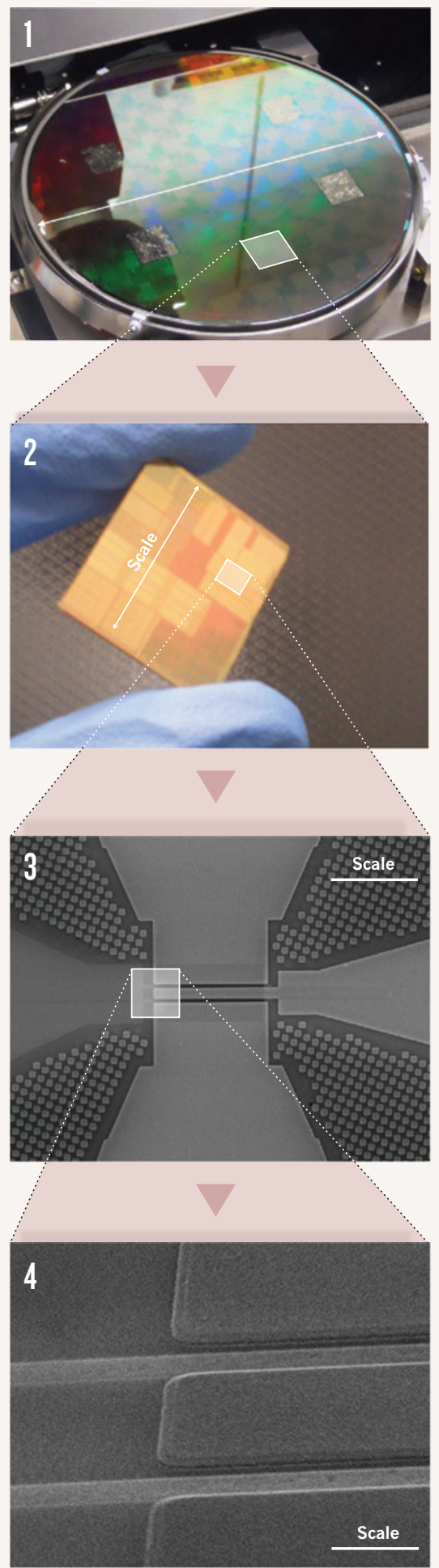

graphene-based analogue electronics are under way at HRL Laboratories in Malibu, California, and at several universities.

Many challenges remain. The IBM frequency mixers won't be found in mobile phones any time soon because the signals they produce are too weak, a problem that originates at the transistor level and that Avouris and others are trying to address. The hundreds of gigahertz speeds reported for graphene devices often ignore an important fact, says Columbia University electrical engineer Ken Shepard. The faster these devices operate, the weaker the signal they generate. When the signal gets overwhelmed by noise, the circuit becomes useless. These effects, dubbed parasitics, mean that in reality, graphene transistors are not much faster than silicon ones. "For these transistors to be earth-changing they have to be at least an order of magnitude better than silicon and that's just not there - they are a factor of two better," Shepard says. Whether these problems are fundamental or are engineering challenges isn't yet clear, he says. "As more designers look at this material, the technology issues will fall away, and we will get a better idea of what the intrinsic limitations are."

\section{EMBRACING ECCENTRICITY}

Rather than try to engineer graphene for highfrequency devices that might one day supplant silicon-based electronics, some researchers are trying to beat new paths. Over the past couple of years, says MIT's Palacios, "people are focusing more and more on applications for graphene that silicon cannot do".

Graphene is stretchy, flexible, transparent and strong; silicon is none of these things. Because graphene has other attractive properties, the material doesn't have to beat silicon to be useful for digital or analogue electronics, says the University of Texas's Akinwande. He believes graphene is suited for applications such as ubiquitous computing or flexible, transparent portable electronics that don't break when dropped. Here the material is competing with very-low-electron-mobility materials such as semiconducting polymers. "The goal of flexible electronics is to make them waterproof, shockproof and environmentally friendly," says Akinwande.

Akinwande is working on making graphene circuits on plastic. The challenges are in the manufacturing, he says. Compared to the smooth surface of a silicon wafer, plastic is bumpy and graphene is extraordinarily sensitive to tiny flaws. Akinwande is working to establish partnerships with companies, including $3 \mathrm{M}$, a technology company based in St Paul, Minnesota, to get plastic that's suitable for graphene transistors. So far, graphene-on-plastic transistors have an intrinsic speed of $5 \mathrm{GHz}-$ significantly slower than Duan's 300-GHz transistors, but faster than the best organic polymer transistors, which share graphene's flexibility.

"Graphene will go first into applications that only graphene can serve," says Palacios. He also sees promise for graphene in flexible electronics. And from that day in the airport in 2008 , he's been working on turning his frustration with graphene's lack of a bandgap into an advantage in highly efficient graphene devices: single transistors that do the work of many silicon devices, and do it better. A single graphene device could do the work of " 20 silicon transistors", Palacios says, which would "reduce power consumption and save space".

Graphene transistors can multitask because as the voltage varies, they reach a minimum current and then switch back and forth between carrying negative and positive charges. Kartik Mohanram, an engineer at the University of Pittsburgh in Pennsylvania, likens such ambipolarity to an eccentric water tap. It would be as if when you turn off the cold tap, the flow tapers off before releasing a gush of hot water.

Most engineers avoid graphene's ambipolarity, making sure these devices operate only in voltage ranges that conduct either positive or negative charges, but not both. By varying the voltage on a graphene transistor all along that scale from positive to negative, engineers are able to get a device that performs more functions than a conventional transistor. Palacios says that one of the ambipolar transistors he designed can convert a digital signal into a Bluetooth signal that can be sent between, for example, a mobile phone and a wireless headset. The conversion requires an analogue device called a phase-shift-key modulator. When made in silicon, this kind of modulator requires an integrated circuit chip. A single ambipolar graphene transistor, Palacios has shown, can do the job all by itself.

Mohanram admits that the idea that ambipolarity can have practical uses is not yet widely accepted by electrical "A single
graphene
device could
do the work
of 20 silicon
transistors." engineers. "The big challenge is whether we can do this on a large scale," he says. While circuit designers can come up with a new idea after a week's thought, it can take a year to make and test just a few devices. Engineers are still learning how to work with graphene, and new ideas come along faster than they can be tested.

Ultimately, for graphene to make a big impact in electronics, the material will have to live up to its hype, and then some. As Texas Instruments' Colombo puts it, silicon "is a very tough act to follow". But patience could be the watchword. After all, it was only in 2004 that a large number of researchers began working with the material. "Maybe graphene will revolutionize electronics," says de Heer, the Georgia Tech physicist. "But not tomorrow."

Katherine Bourzac is a science journalist based in San Francisco, California. 